\title{
Examining and Reducing the Influence of Sampling Errors on Feedback-Driven Optimizations
}

\author{
MINGZHOU ZHOU, IBM \\ BO WU, Colorado School of Mines \\ XIPENG SHEN, North Carolina State University \\ YAOQING GAO and GRAHAM YIU, IBM Toronto Lab
}

\begin{abstract}
Feedback-driven optimization (FDO) is an important component in mainstream compilers. By allowing the compiler to reoptimize the program based on some profiles of the program's dynamic behaviors, it often enhances the quality of the generated code substantially. A barrier for using FDO is that it often requires many training runs to collect enough profiles to amortize the sensitivity of program optimizations to program input changes. Various sampling techniques have been explored to alleviate this time-consuming process. However, the lowered profile accuracy caused by sampling often hurts the benefits of FDO.

This article gives the first systematic study in how sampling rates affect the accuracy of collected profiles and how the accuracy correlates with the usefulness of the profile for modern FDO. Studying basic block and edge profiles for FDO in two mature compilers reveals several counterintuitive observations, one of which is that profiling accuracy does not strongly correlate with the benefits of the FDO. A detailed analysis identifies three types of sampling-caused errors that critically impair the quality of the profiles for FDO. It then introduces a simple way to rectify profiles based on the findings. Experiments demonstrate that the simple rectification fixes most of those critical errors in sampled profiles and significantly enhances the effectiveness of FDO.
\end{abstract}

Categories and Subject Descriptors: D.3.4 [Programming Languages]: Optimization

General Terms: Compiler, Profiling, Feedback-Driven Optimization (FDO), Performance, Input Sensitivity

Additional Key Words and Phrases: Performance, influence of sampling errors, feedback-driven optimization

This material is based on work supported by the DOE Early Career Award and the National Science Foundation (NSF) under Grant Nos. 1455404 and 1525609 and the Career Award, IBM CAS Fellowship, and Google Faculty Award. Any opinions, findings, and conclusions or recommendations expressed in this material are those of the authors and do not necessarily reflect the views of the DOE, the NSF, IBM, or Google.

This articles extends an ECOOP'13 conference paper [Wu et al. 2013] by the same authors in several major aspects. First, it introduces a new type of error, inequality errors, in addition to zero errors and equality errors that have been presented in ECOOP'13. More importantly, it reveals that a class of inequality errors, called branch bias errors, are especially crucial to FDO performance (Section 3.1.3). Second, it analyzes the relations among the three major types of sampling-caused errors (Section 3.1.4) and reports the influence of those errors on profile accuracy (Section 3.1.5). Third, it presents an enhanced design of profile rectification that leverages the newly introduced branch bias errors and their relationship with other types of errors, and reports the updated performance results (Section 3.2). In addition, the presentation throughout the article has been substantially enhanced and more references have been added.

Authors' addresses: M. Zhou, 11501 Burnet Road, Austin, TX 78758; email: mzhou@us.ibm.com; B. Wu, Electrical Engineering \& Computer Science, Colorado School of Mines, Golden, CO 80401; email: bwu@mines.edu; X. Shen, Computer Science Department, North Carolina State University, Raleigh, NC 27695; email: xshen5@ncsu.edu; Y. Gao and G. Yiu, 8200Warden Ave, IBM Toronto Lab, Markham, ON, Canada, L6G 1C7; emails: \{ygao, gyiu\}@ca.ibm.com.

Permission to make digital or hard copies of part or all of this work for personal or classroom use is granted without fee provided that copies are not made or distributed for profit or commercial advantage and that copies show this notice on the first page or initial screen of a display along with the full citation. Copyrights for components of this work owned by others than ACM must be honored. Abstracting with credit is permitted. To copy otherwise, to republish, to post on servers, to redistribute to lists, or to use any component of this work in other works requires prior specific permission and/or a fee. Permissions may be requested from Publications Dept., ACM, Inc., 2 Penn Plaza, Suite 701, New York, NY 10121-0701 USA, fax +1 (212) 869-0481, or permissions@acm.org.

(c) 2016 ACM 1544-3566/2016/04-ART6 $\$ 15.00$

DOI: http://dx.doi.org/10.1145/2851502 
ACM Reference Format:

Mingzhou Zhou, Bo Wu, Xipeng Shen, Yaoqing Gao, and Graham Yiu. 2016. Examining and reducing the influence of sampling errors on feedback-driven optimizations. ACM Trans. Archit. Code Optim. 13, 1, Article 6 (April 2016), 24 pages.

DOI: http://dx.doi.org/10.1145/2851502

\section{INTRODUCTION}

Feedback-driven optimization (FDO) is an important technique for modern compilers to enhance program performance. By collecting behaviors such as basic block frequencies and frequently called functions from some training runs, FDO provides better heuristics to help compilers optimize programs. Experiments have shown that FDO often brings significant extra speedups to programs beyond what static compilations provide. It has become an important component of modern compilers, exemplified by GCC, IBM XLC, and Intel ICC.

A barrier for practical usage of FDO is the overhead in collecting profiles of program executions. The overhead ranges from $20 \%$ to as much as $100 \times$ [Shen et al. 2007] (when data accesses are profiled) of the execution time of the original program, depending on the types of profiles to collect. Figure 1 shows that on an IBM XLC compiler (v12.1), the programs with profile collection (detailed in Section 2.1) run $5.4 \times$ (up to $22 \times$ ) slower than their default runs. It is especially a concern for long-running programs and is underscored by the increasing need for data analytic applications that often handle data in a great variety in formats, sources, values, and patterns (one of the key properties of Big Data). As many studies have shown, different input datasets often demand different optimization decisions [Tian et al. 2010; Berube et al. 2011]. To make code generated by FDO able to adapt to various datasets, it is necessary to collect a large number of profiles on different input datasets [Jiang et al. 2010]. The needed long profiling time impairs the practical value of FDO.

A solution that has been explored before is sampling. It collects profiles on only a small portion of the program execution, with the hope that the compiler can still find out important behavior patterns from the sampled profile and apply FDO effectively. However, sampling unavoidably introduces some inaccuracy into the collected profiles, which may impair the exertion of the full power of FDO in turn. There have been some studies on reducing biases in sampling [Mytkowicz et al. 2010] and some proposals of improved sampling schemes, such as bursty sampling [Mousa and Krintz 2005; Arnold and Ryder 2001; Hirzel and Chilimbi 2001; Arnold and Grove 2005]. Although these techniques can improve the profile's quality to a certain degree, the speedup by FDO on the sampled profiles still has a substantial gap from what it produces on full profiles, as Figure 2 shows.

In this work, we address this problem from a different perspective: instead of refining sampling schemes, we attempt to rectify errors in a sampled profile after it is collected. We call this new approach profile rectification.

Some fundamental questions need to be answered before an effective rectification method can be designed. In particular, two questions are critical: What are the relations among sampling rates, accuracy of the collected profile, and its usefulness for FDO? How do the errors in a profile influence the optimizations and how can the critical errors be rectified? Answers to these questions are essential for guiding the directions of profile rectification. But to the best of our knowledge, none of these questions have been systematically studied on modern compilers and systems.

To answer these open questions, we conduct a twofold investigation. First, as shown in Section 2, we design a set of systematic measurements to reveal the statistical correlations among sampling rates, profile accuracy, and the corresponding FDO benefits. We focus on the FDO in two mature compilers, IBM XLC compiler and GCC, and 


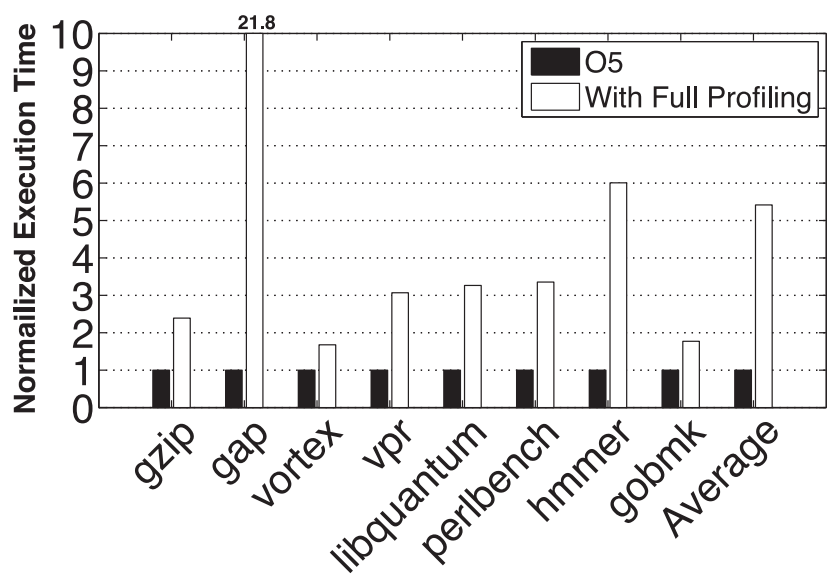

Fig. 1. Profile collection time, normalized by the default execution time of the programs.

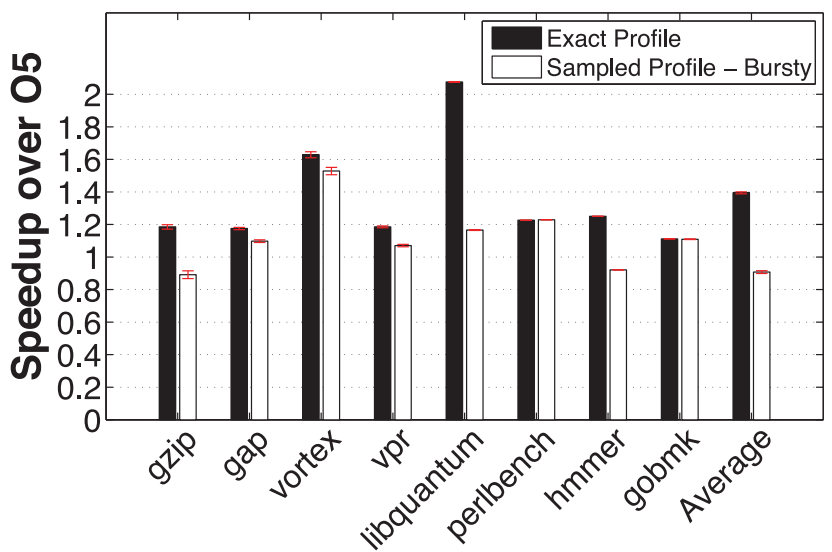

Fig. 2. Speedups (compared to static compilation with the highest-level optimizations enabled) produced by the FDO of IBM XLC (v12.1) compiler when sampled (bursty at rate of 5\%) and when full profiles are used, respectively. The error bars show the $95 \%$ statistical confidence intervals.

concentrate on the basic block frequency profile and edge frequency profile, which are the most critical parts of the profiles used by the two FDO systems. To avoid biases in the analysis, we conduct 7,680 runs, which cover the seven most important factors in four levels, including the usage of the two mature compilers, two sampling methods with six sampling rates for each, two platforms, eight SPEC benchmarks with some nontrivial FDO potential, four inputs per benchmark, and 10 repetitions for each setting. The systematic measurements reveal some counterintuitive observations. It is commonly perceived that a higher sampling rate tends to give more accurate profiles, which would help FDO produce code that has a better performance. However, the experiment results suggest that even though, in general, a higher bursty sampling rate leads to a more accurate profile, this perception isn't true for uniform sampling, which samples one instruction after every fixed time period. Moreover, the results show that for both types of sampling methods, when a more accurate profile is given to the FDO, it often does not produce code with a better performance. In other words, in the sampling rate range, profile accuracy does not have an apparent correlation with the FDO benefits. 
These surprising observations prompt a deep analysis of the influence cast on FDO by various types of sampling errors (Section 3). We investigate three types of errors that potentially have some strong influence. The first is 0 -counter errors, which refer to the case when a counter in the sampled profile is zero but its value in the full profile is not. The second is equality errors, which refer to the case when two counters in the same function have different values in the sampled profile but have the same value in the full profile. The third is inequality errors, which refer to the case when one counter is greater than another in the sampled profile but smaller in the full profile. For the third type, after a series of explorations, we concentrate on a special class of inequality for its special importance. That class is called biased branch errors, which refer to conditional branches that are strongly biased (one branch has a taken rate no smaller than $80 \%$ ) in the full profile but not in the sampled profile.

Our analysis shows that although these types of errors do not affect the overall profile accuracy much, they change the optimization decisions of the FDO dramatically. We then propose to rectify the three types of errors by applying counter value patterns derived from some training profiles. The simple rectification turns out to boost the usefulness of the sampled profiles for FDO, increasing the average speedup from $1.15 \times$ to $1.36 \times$, around $99 \%$ of what full profiles can yield.

In summary, this work makes three main contributions:

-Correlations. It is the first study that systematically uncovers the correlations among sampling rates, profile accuracy, and the usefulness for FDO.

-Influence of Errors. It offers a set of novel insights on sampling and its influence on FDO:

- The 0-counter and equality errors in sampled profiles impair FDO substantially.

-A general rectification of inequality errors adds no extra benefits over 0 -counter and equality error rectifications, but biased branch error rectification does. In fact, the best results are obtained when 0 -counter errors and biased branch errors are rectified.

-Uniform sampling not only underperforms bursty sampling but also shows a weak correlation between sampling rate and profile accuracy, which reinforces the superiority of bursty sampling over uniform sampling for FDO.

-Commonly defined accuracy, either weighted or unweighted, fails to quantify the actual quality of a profile for FDO.

-Profile Rectification. To the best of our knowledge, this is the first work showing that simple profile rectification can dramatically enhance the usefulness of a profile for FDO. The proposed profile rectification provides a simple and practical way to enhance the effectiveness of sampling-based FDO.

The rest of the article is organized as follows. In Section 2, we first briefly introduce the two FDO compilation systems, the two sampling methods, and some other experiment settings. Then we present the findings of the correlations. In Section 3, we present a deep analysis of the sampling errors and describe the simple profile rectification method. Section 4 discusses the implications of the findings to FDO compilers of traditional imperative languages and to the runtime of managed languages (e.g., Javascript, Java, Python) that adopt online sampling and Just-In-Time (JIT) compilation. We discuss some related work in Section 5 and then conclude the article with a summary.

\section{COUNTER INTUITIVE CORRELATIONS}

This section starts with an introduction to the FDO in the two compilers we use. It then presents the design of the empirical measurements and reports the findings on the correlations among sampling, profiles, and their usefulness. 
Table I. Dimensions Covered in the Experiment Design

\begin{tabular}{l|c|c|l}
\hline Levels & Dimensions & Variations & \multicolumn{1}{c}{ Description } \\
\hline \multirow{2}{*}{ workload } & benchmarks & 8 & SPEC CPU2000 \& CPU2006 \\
\cline { 2 - 4 } & inputs & 4 & 1 train input, 3 ref inputs \\
\cline { 2 - 4 } & compilers & 2 & XLC, GCC \\
\cline { 2 - 4 } & platforms & 2 & Intel Xeon \& IBM POWER7 \\
\cline { 2 - 4 } & methods & 2 & Bursty, uniform \\
\cline { 2 - 4 } & frequencies & 6 & 6 for bursty, 6 for uniform \\
\hline
\end{tabular}

\subsection{Background on FDO}

FDO is part of many modern compilers. The implementations of FDO in different compilers may differ in what set of optimizations they contain but mostly follow a similar high-level design. We briefly describe the way FDO works in a mature commercial compiler, IBM XLC, as follows.

To enable FDO, two stages of compilations are necessary. For XLC, in the first stage, the compiler must be invoked with a special option ("-qpdf1"). With that option, the compiler does detailed instrumentation to the program such that after an execution of the program, the exact execution frequency of every basic block (along with other information) can be obtained. We call the file containing all these exact profiling results an exact profile. A basic instrumentation scheme is that the compiler inserts some instructions into every basic block such that after an execution of the program, the exact execution frequency of every basic block is obtained. The XLC compiler avoids inserting such instructions into a basic block if its frequency can be exactly inferred from the frequencies of the already-instrumented basic blocks. In the second stage, the compiler is invoked again with another special option (“-qpdf2"). In this round of compilation, the compiler enables FDO, which reads the profile and produces an optimized executable.

As the profiles capture some runtime behaviors (e.g., the hotness of a function or basic block), they can provide the optimizers some hints that static code analysis is unable to provide. FDO heavily exploits those hints to enhance code layout, inline functions, and so on. An inaccurate profile may hence mislead FDO into making wrong optimization decisions.

\subsection{Experimental Design}

We design a set of experiments to empirically measure the relations among sampling rate, profile accuracy, and the influence on the effectiveness of FDO. In the design, we carefully cover seven dimensions that are closely relevant to the relations to minimize the bias in the measurement. They fall into four levels, as summarized by Table I and explained as follows.

2.2.1. Benchmarks and Inputs. Given that the focus of this work is on FDO, when choosing benchmarks, we concentrate on those that exhibit some nontrivial speedups when FDO is applied. Meanwhile, our current infrastructure works on C programs only. Our observations show that integer programs pose major challenges for static analysis and optimizations for their complex control flows and large number of functions. They hence exhibit more potential for FDO than floating-point programs do. We examined all C integer programs in SPEC CPU2000 and CPU2006; Table II reports the speedups from FDO when the exact profiles are used. The top eight programs, for their substantial FDO potential (>10\% speedups), are chosen to be used in this study. (We have observed that sampled profiles typically give less FDO speedup for the loss of information caused by the sampling; there are some exceptions, but overall, the top eight programs do show 
Table II. Benchmarks and FDO Speedup from Exact Profiles over O5 Compilation

\begin{tabular}{|l|c|c|c|}
\hline Program & Benchmark Suite & Description & FDO Speedup \\
\hline gzip & CPU2000 & Compression & $\mathbf{1 . 1 9} \times$ \\
\hline gap & CPU2000 & Group Theory, Interpreter & $\mathbf{1 . 1 8} \times$ \\
\hline vortex & CPU2000 & Object-Oriented Database & $\mathbf{1 . 6 3 \times}$ \\
\hline vpr & CPU2000 & FPGA Circuit Placement and Routing & $\mathbf{1 . 1 9 \times}$ \\
\hline libquantum & CPU2006 & Quantum Computing & $\mathbf{2 . 0 9 \times}$ \\
\hline perlbench & CPU2006 & Perl Interpreter & $\mathbf{1 . 2 2 \times}$ \\
\hline hmmer & CPU2006 & Search Gene Sequence & $\mathbf{1 . 2 5 \times}$ \\
\hline gobmk & CPU2006 & Artificial Intelligence & $\mathbf{1 . 1 1} \times$ \\
\hline crafty & CPU2000 & Game Playing: Chess & $1.08 \times$ \\
\hline parser & CPU2000 & Word Processing & $1.06 \times$ \\
\hline twolf & CPU2000 & Place and Route Simulator & $1.07 \times$ \\
\hline gcc & CPU2006 & C Compiler & $1.07 \times$ \\
\hline bzip2 & CPU2006 & Compression & $1.09 \times$ \\
\hline sjeng & CPU2006 & Artificial Intelligence: Chess & $1.02 \times$ \\
\hline mcf & CPU2006 & Combinatorial Optimization & $1.07 \times$ \\
\hline h264ref & CPU2006 & Video Compression & $1.07 \times$ \\
\hline
\end{tabular}

the largest potential for FDO among all the programs in our observations.) As Figure 2 shows, these benchmarks show an average $1.39 \times$ speedup when FDO is applied (on the exact profiles of the execution inputs) compared to their performance through static compilations using XLC.

For each program, besides including both its train and ref inputs coming with the benchmark suite, when necessary, we collect or create two extra representative inputs by searching for the real usage of their original applications or reading the source code. The extra inputs are used in the experiments described in Section 3 for examining the stableness of profile value patterns across different inputs. For FDO, the profiles are collected on the train input and evaluated on the ref input.

2.2.2. Compilers. We select the recent versions of XLC (v12.1) and GCC (v5.1) as our compilers. Both compilers have been developed for more than a decade. The former is the main commercial compiler of IBM for $\mathrm{C}$ and $\mathrm{C}++$ and shares the core with many other IBM compilers for other languages. Its FDO is sophisticatedly polished by a large compiler team for many years, able to exploit profiles to conduct a number of advanced intraprocedure and interprocedure optimizations. GCC is a result of the many years of efforts by the open-source community. Its performance has been shown to get close to commercial compilers in many cases. Its FDO component has also been developed for quite a while. We use both compilers for this study to examine the influence of different FDO implementations on the studied relations.

The type of profile collected by XLC is a basic block frequency profile, where each counter records the number of times a basic block is encountered in an execution. From that profile, XLC could derive the control flow edge profile and the function calling edge profile of that execution, which record the frequency of each edge in the control flow graphs and the frequency of each edge in the call graphs of the program. In the instrumentation stage of XLC, each procedure's control flow graph is explored to find out some straight lines of basic blocks that must have the same counter value. Only the first basic block of a straight line needs to be instrumented to collect access frequency. During recompilation, function calling frequencies and control flow branch probabilities are inferred from basic block counters and the mapping, serving as hints for the FDO. A similar implementation scheme is shown in GCC. It collects control flow 
edge profiles and derives the other types of profiles, and differences exist in the set of optimizations they include and how those optimizations are implemented.

2.2.3. Platforms. We run XLC-related experiments on an IBM Power7 machine, which has the AIX 7 operating system installed. We conduct the GCC-related experiments on a machine equipped with Intel Xeon W3550, running an OpenSUSE Linux, version 12.1.

2.2.4. Sampling Methods and Frequencies. We experiment with two sampling methods. The first is uniform sampling, which is the most commonly used sampling method. It tries to get a sample after a given time interval. For example, when being applied to collect basic block frequency profiles, the runtime sampler checks which instruction is being executed and finds out which basic block that instruction belongs to after a given time interval; it then increases the counter corresponding to the basic block by one. The second sampling method is bursty sampling. In this method, there are two predefined parameters, the execution period length $\tau_{e}$ and the profiling period length $\tau_{p}$, which can be in some time unit like the number of instructions (as used in this work). The runtime switches between normal execution and profiled execution periodically. During an execution, after a $\tau_{e}$-long period of normal execution, the runtime switches the execution to a fully instrumented version and runs that version for a $\tau_{p}$-long period of time to collect some profiles, and then switches back to normal execution. The backand-forth switching continues throughout the program execution.

For bursty sampling, we modify the instrumentation, so one execution directly produces one sampled profile. For uniform sampling, based on the full profile and the size of each basic block, we find out the total number of dynamic instructions (block size multiplied by the frequency of the block) a basic block corresponds to, denoted as $n_{i}$ for block $i$, and the total number of instructions overall, denoted as $N$. If the sampling rate is $r$, we sample $N * r$ times. Each time, there is $n_{i} / N$ probability for block $i$ to be sampled, and the counter of the sampled block increases by one.

Previous studies have shown that the bursty sampling, although being more complicated to implement, can often produce a more accurate profile than the uniform sampling does at the same sampling rate [Arnold and Grove 2005]. Bursty sampling has been implemented in some runtime systems, such as Jikes RVM [Arnold and Ryder 2001]. Using both sampling methods helps us examine the influence of different sampling schemes on the relations between profile accuracy and FDO.

We experiment with six sampling rates for each of the sampling methods. These rates subsume the typical range of sampling rates used in practical systems. The sampling rate of uniform sampling is determined by a single parameter, the sampling period length $\left(10^{2}, 10^{3}, 10^{4}, 5 * 10^{4}, 10^{5}, 5 * 10^{5}\right)$. Because the bursty sampling has two parameters, the execution period length $\tau_{e}$ and profiling period length $\tau_{p}$, each of its sampling rates is represented with the ratio of a pair, $\tau_{p} /\left(\tau_{e}+\tau_{p}\right)$. The rates are $1 / 1000$, $10 / 1000,50 / 1000,100 / 1000,200 / 1000$, and 400/1000. The sampling rates for uniform sampling reside in a similar range.

2.2.5. Time Measurement. In all runs, the highest optimization level is enabled. We see some minor fluctuations (less than $8 \%$ ) in the execution times of multiple runs in the same setting. But still, to minimize the influence of random noise, we repeat each run 10 times and report the average. Harmonic mean [Eeckhout 2010] is used in computing the average speedup across benchmarks.

\subsection{Measurements and Findings}

The coverage of the various factors leads to 7,680 runs in total. This subsection presents the findings we have obtained from these measurements. But before that, we first explain some metrics we use to quantify profile accuracy and correlations. 
2.3.1. Accuracy Metrics. Let $B_{i}$ represent the exact profile (or full profile) of a run on input $i$. Exact profiles can be obtained through a full profiling. Let $S P_{i}^{\prime}$ be the profile obtained by sampling. Before comparing the two profiles, we multiply each counter in $S P_{i}^{\prime}$ by the ratio between the sum of the counters in $B_{i}$ and that in $S P_{i}^{\prime}$ so that the two profiles are at the same scale for comparison. We denote the scaled sampled profile with $S P_{i}$. We use $S P_{i}[j]$ and $B_{i}[j]$ to denote the counter values of the $j$ th item in the sampled and exact profiles, respectively. For the purpose of explanation, we use basic block frequency profiles as our example in the following discussion. In such a profile, each item corresponds to the frequency of a basic block being accessed.

The definition of the accuracy should quantify the similarity between $S P_{i}$ and $B_{i}$. We define the accuracy (Acc) of a basic block counter as follows:

$$
A c c_{i}[j]=1-\frac{\left|S P_{i}[j]-B_{i}[j]\right|}{\max \left(S P_{i}[j], B_{i}[j]\right)} .
$$

The use of the "absolute" operator and $\max$ is to normalize the accuracy to the range of $[0,1]$. (Note, since $S P_{i}$ is the sampled profile after scaling, it is possible that $S P_{i}[j]$ becomes greater than $B_{i}[j]$.) We use two definitions for the overall accuracy of a profile. An unweighted accuracy (UAcc) is just an arithmetic average of all basic blocks' accuracies. It treats each basic block equally. A weighted accuracy (WAcc) of a profile is a weighted average as follows:

$$
W_{A c c_{i}}=\sum_{j} A c c_{i}[j] \times \frac{B_{i}[j]}{\sum_{j} B_{i}[j]},
$$

where the weights are proportional to the significance of a basic block in the program in terms of its access frequency.

2.3.2. Correlation Metrics. Among the different variations of commonly used correlations metrics, we find the Spearman's rank correlation coefficient (called rank coefficient in short) suiting our needs. Let $X$ and $Y$ represent two ordered lists of values. The order is based on some predefined criterion (e.g., an ascending order of the values of the elements). The position of an element in the ordered list is called the rank of that element. The rank coefficient is defined as follows:

$$
r=\frac{\sum_{i}\left(x_{i}-\bar{x}\right)\left(y_{i}-\bar{y}\right)}{\sqrt{\sum_{i}\left(x_{i}-\bar{x}\right)^{2} \sum_{i}\left(y_{i}-\bar{y}\right)^{2}}},
$$

where $x[i]$ and $y[i]$ are the ranks of $X[i]$ and $Y[i]$ in $X$ and $Y$, respectively.

Recall that the questions we try to answer are whether a higher sampling rate leads to a more accurate profile and hence more benefits from FDO. The rank coefficient fits our needs as it assesses how well the relationship of two variables fits in a monotonic function. In comparison, the standard Pearson coefficient measures whether two variables form a linear relation, which is a property unnecessarily stronger than what we need.

The value of a rank coefficient is always between -1 and 1 , with a value close to 1 implying a strong coincreasing relation between $X$ and $Y$, and a value close to -1 implying that the two variables' values are taking an opposite trend.

2.3.3. Sampling Rate and Profile Accuracy. Figures 3 and 4 report the weighted and unweighted accuracies of the sampled profiles of all benchmarks when different sampling rates are used. The profiles for the bursty sampling have a close-to-perfect weighted accuracy across all sampling rates, while the profiles for the uniform sampling have an average $64 \%$ accuracy. The intuition behind the large accuracy disparity is that because each time the uniform sampling checks only one instruction, a larger basic block 


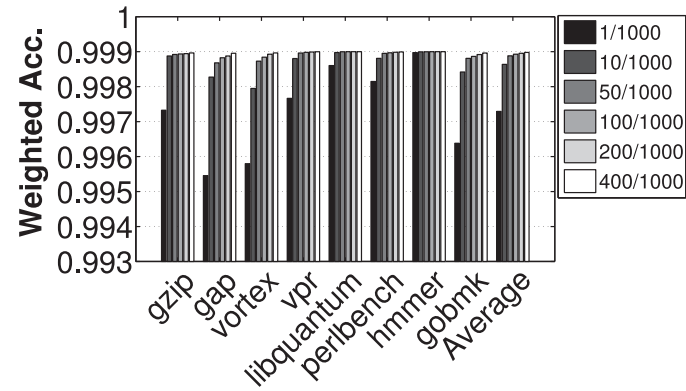

(a)

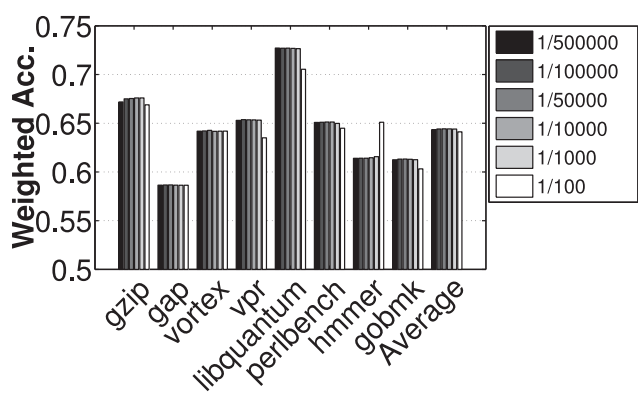

(b)

Fig. 3. (a) Weighted accuracy of bursty sampling. (b) Weighted accuracy of uniform sampling.

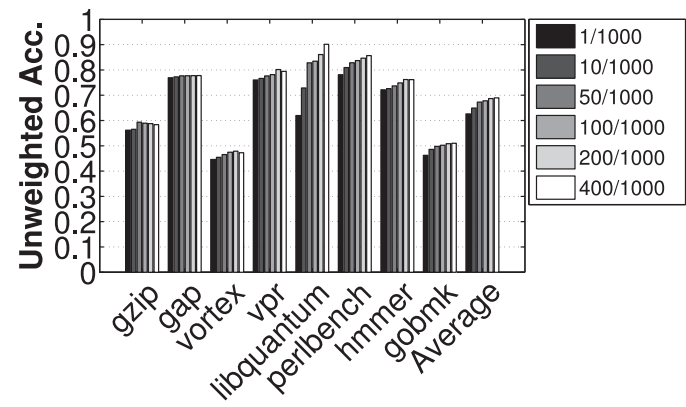

(a)

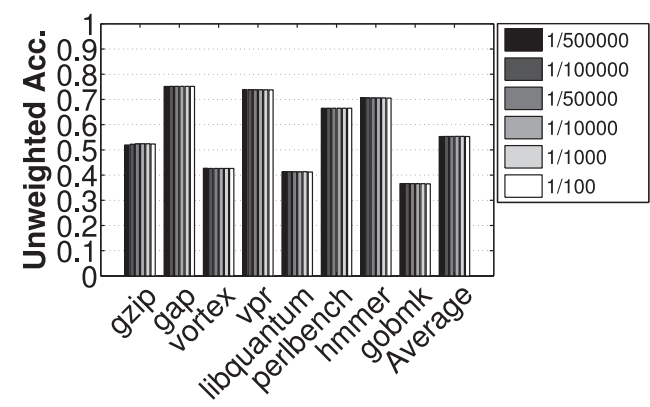

(b)

Fig. 4. (a) Unweighted accuracy of bursty sampling. (b) Unweighted accuracy of uniform sampling.

Table III. Rank Correlation Coefficients Between Sampling Frequency and Profile Accuracy

\begin{tabular}{|l|c|c|c|c|}
\hline Program & Weighted Bursty & Unweighted Bursty & Weighted Uniform & Unweighted Uniform \\
\hline gzip & 1 & 0.43 & 0.09 & 0.66 \\
\hline gap & 1 & 1 & -0.6 & 0.9 \\
\hline vortex & 1 & 0.83 & -0.03 & -0.94 \\
\hline vpr & 1 & 0.94 & -0.43 & -0.37 \\
\hline libquantum & 1 & 1 & -0.94 & 0.086 \\
\hline perlbench & 1 & 1 & -0.49 & -0.6 \\
\hline hmmer & 1 & 0.94 & 1 & -0.66 \\
\hline gobmk & 1 & 1 & -0.37 & -0.43 \\
\hline Median & 1 & 0.97 & -0.4 & -0.4 \\
\hline
\end{tabular}

gets a larger chance to be sampled than a smaller basic block does if the two blocks actually have the same frequencies of being executed. The issue is less serious in bursty sampling. For bursty sampling, block size may cast some influence on which block the sampling period starts from, but the influence is much weaker to the overall accuracy because within a sampling period, the size of a basic block less affects the chance for it to get sampled. These results echo some previous observations on the two sampling methods [Hirzel and Chilimbi 2001; Arnold and Grove 2005]. The unweighted accuracy difference is smaller, but bursty sampling still outperforms uniform sampling in general.

Table III provides the rank coefficients between sampling rates and profile accuracies. When weighted accuracy is used, the coefficients are all one for bursty sampling, indicating the very strong correlation between sampling frequency and profile accuracy. In other words, the profile accuracy will definitely increase when we use a higher 


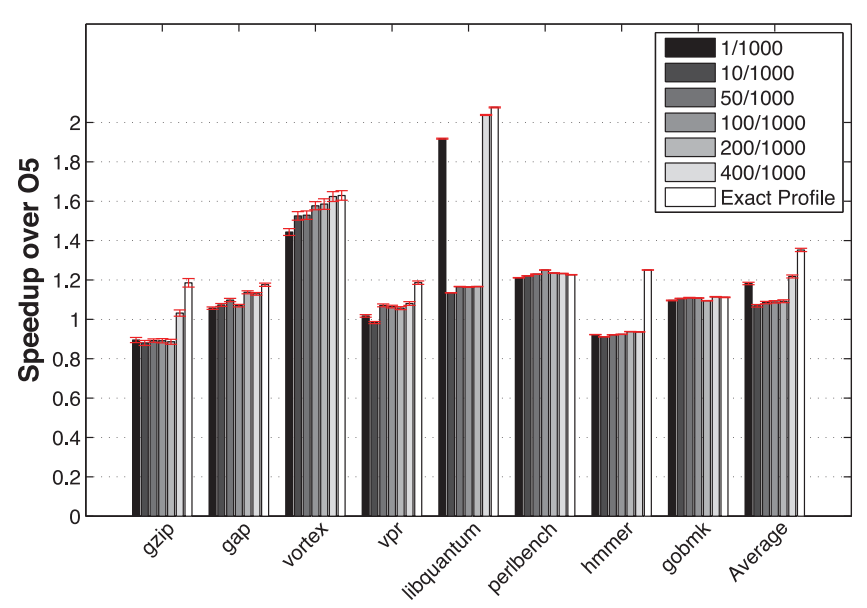

Fig. 5. Speedup comparison between sampled profiles of six sampling rates (1/1000, 10/1000, 50/1000, 100/1000, 200/1000, and 400/1000) and exact profiles.

sampling rate. Visually, despite the high accuracies from all sampling rates, such a relation can still be consistently seen from the small differences among the bars in Figure 3(a). When unweighted accuracy is used, the correlations are slightly lower, but still close to one for most programs, as Figure 4(a) shows.

Uniform sampling shows much weaker correlations with profile accuracy. Visually, from Figures 3(b) and 4, we see no apparent correlations between the sampling rates and the accuracy of the uniformed sampled profiles. A higher rate of uniform sampling does not lead to a more accurate profile. Quantitatively, the average of the rank coefficient is only -0.22 when weighted accuracy is used. Two programs, hmmer and libquantum, are exceptions. For hmmer, a higher sampling rate leads to more accurate profiles, while for libquantum, the trend is the opposite. The reason for the weak correlations comes from the same source (the effects of basic block sizes) for the low-profile accuracy mentioned earlier in this section. To further understand the severity of the effects, we extend the sampling rate to some large values $(20 \%, 40 \%, 80 \%)$ that are rarely used in actual runtime sampling. The results show that even at such a level of sampling rates, the correlations are not much stronger than what Table III has shown.

These results provide two insights. First, they confirm and further reinforce that bursty sampling is more suitable for program profiling than uniform sampling. As Figures 3 and 4 show, even when the bursty sampling uses the lowest sampling frequency (1/1000), it still outperforms the uniform sampling at all its sampling frequencies including the one (1/100) that is 10 times higher than 1/1000. Second, the weak correlations of uniform sampling suggest that the shortcoming of uniform sampling for program profiling is deeply inherent in the method and can hardly be overcome by an increase in sampling rate. Given that uniform sampling is still the most commonly used runtime profiling method in today's systems, these insights hopefully will prompt developers to revisit the sampling methods they select.

2.3.4. Profile Accuracy and FDO Benefits. Figure 5 reports the speedups FDO produces on the full profiles and profiles collected through bursty sampling at three sampling rates. Recall that for bursty sampling, higher sampling rates always lead to more accurate profiles. However, the bars in Figure 5 show a quite irregular pattern in the speedups as the sampling rate increases. While gap, vortex, and perlbench follow the intuitive trend of benefiting more from more accurate profiles, all the other benchmarks show an opposite trend sometimes-degraded performance from more accurate profiles. The 
Table IV. Rank Correlation Coefficients Between Profile Accuracy and Performance

\begin{tabular}{|l|c|c|c|c|}
\hline Program & Weighted Bursty & Unweighted Bursty & Weighted Uniform & Unweighted Uniform \\
\hline gzip & -0.14 & -0.29 & -0.15 & 0.58 \\
\hline gap & 0.75 & 0.75 & -0.85 & 0.34 \\
\hline vortex & 0.88 & 0.59 & 0.08 & -0.8 \\
\hline vpr & 0.62 & 0.79 & -0.01 & -0.07 \\
\hline libquantum & -0.08 & -0.08 & -0.5 & 0.16 \\
\hline perlbench & 0.82 & 0.82 & 0.51 & 0.63 \\
\hline hmmer & 0.47 & 0.41 & 0.11 & -0.76 \\
\hline gobmk & 0.41 & 0.41 & -0.42 & -0.59 \\
\hline Median & 0.55 & 0.5 & -0.08 & 0.05 \\
\hline
\end{tabular}

extreme case on libquantum even showed $75 \%$ more speedup from the lowest sampling rate than from the highest sampling rate. Overall, the FDO effect from the exact profiles is the best, by providing $14 \%$ more speedup than the best sampling rate.

Table IV reports the rank coefficients between profile accuracies and the speedups. No benchmarks have near 1 correlation coefficient. Only two benchmarks (vortex, perlbench) have coefficients larger than 0.8 on bursty sampling when weighted accuracy is used. So for them, higher bursty sampling rates are likely to bring better optimizations. But for most benchmarks, there is only weak or no correlation between profile accuracy and the usefulness for FDO. The program gap even has a coefficient of -0.85 on uniform sampling, indicating a largely monotonic decreasing relation between profile accuracy and usefulness for FDO.

Short Summary. Current FDO optimization systems are constructed mostly on a common perception that larger sampling rates tend to lead to better performance. This section debunks the intuition by first showing that a higher sampling frequency does not necessarily give us more accurate profiles, and the effect depends on the sampling method. This indicates that we should be more careful about the design of the sampler. More surprisingly, we show that there are very weak correlations between the accuracy of a profile and its usefulness for FDO, no matter which sampling method is used. It does not mean that we can just feed the compiler with randomly generated profiles for good FDO-driven performance. As the results show, the best performance mostly still comes from the exact profiles for most benchmarks. The findings suggest that current understanding to how profiling errors influence FDO is preliminary; some deep analysis into the results is necessary, as given in the next section.

\section{DEMYSTIFICATION AND PROFILE RECTIFICATION}

The previous section showed that for most benchmarks, there exists only very weak correlations between profile accuracy and its usefulness for FDO. However, we observe that sampled profiles do not perform as well as exact profiles, which means sampling errors do play an important role. After analyzing the influence of various types of errors, we identify three kinds of sampling errors that have some important effects on FDO benefits: 0 -counter errors, equality errors, and inequality errors. In this section, we first present some analysis results on how these kinds of errors impair the effectiveness of FDO, and then show that they can be fixed through a simple profile rectification, and finally report the significant speedup increment the rectification helps FDO generate.

\subsection{Deep Analysis on Profile Errors}

3.1.1. 0-Counter Errors. The first type of error is 0-counter errors, referring to the case when a counter in a sampled profile equals zero but its value in the full profile is not. For the purpose of explanation, we will concentrate our discussion on basic block frequency profiles. 


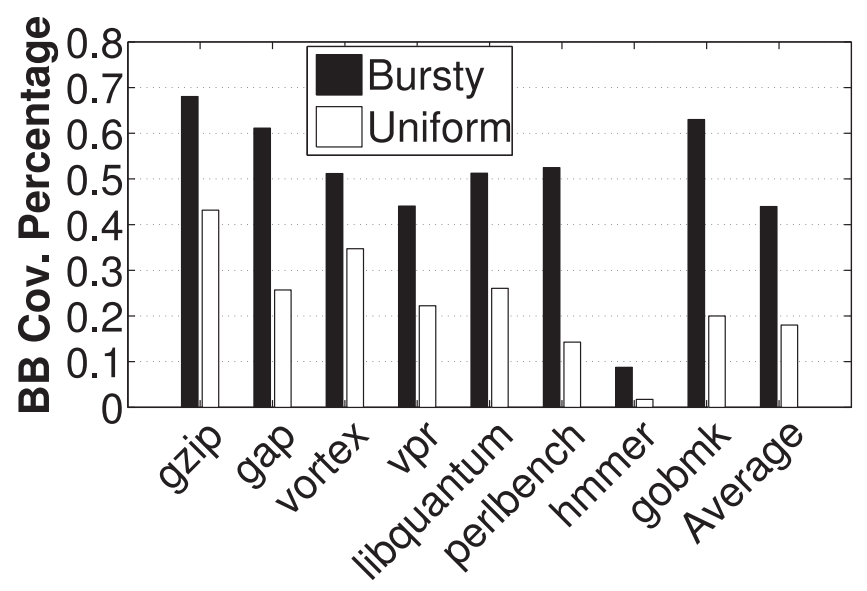

Fig. 6. Basic block coverage comparison between exact profiles and sampled profiles.

Sampling, by nature, misses some parts of a program execution. But basic blocks that have a small value in the exact profile are especially easy to be missed completely by the sampler. To get an intuition of how likely this can be, we give a simple analysis for an example scenario. Consider a program whose basic blocks are similar in size so that in the sampling scheme, every instance of its blocks has the same probability to get sampled. If the counter of a block $B B_{i}$ is $k$ in the exact profile and the bursty sampling samples every $m$ out of $n$ basic block instances, and assuming the chance for a block to be executed is the same throughout a program execution, the probability for $B B_{i}$ to be sampled at least once is $\left(1-\left(1-\frac{m}{n}\right)^{k}\right)$. If $m=1, n=1,000$, the probability for $B B_{i}$ to be sampled at all is less than $1 \%$ if $k<10$. Given the $20-80$ rule (i.e., commonly $20 \%$ of a program is responsible for about $80 \%$ of its execution), most basic blocks are relatively cold, and hence have some good probabilities to get missed by the sampler, causing 0 -counter errors.

Figure 6 shows the basic block coverage of the sampled profiles. ${ }^{1}$ The coverage is defined as the percentage of the nonzero counters in the exact profile that also have nonzero values in the sampled profile. This metric shows how well the sampled profile represents the coverage pattern of the exact profile. We observe an average of $56 \%$ basic block coverage reduction by bursty sampling. In the worst case shown on $\mathrm{hmmer}$, more than $92 \%$ of basic blocks are completely missed by the sampler. The coverage by uniform sampling is even worse, only $18 \%$.

Through a detailed analysis of the influence of 0-counter errors on the various optimizations in FDO, we find that two optimizations, function inlining and loop optimizations, are influenced the most. As Section 2.2 mentioned, function calling frequencies are inferred from basic block counters in XLC. If the basic block containing a function call has counter 0 , the recompilation totally ignores the corresponding call edge. If all basic blocks invoking a function have 0 counters, all the profile information of that function is ignored, even if that function takes a substantial amount of execution time (e.g., containing some loops). This implies that the counter values of calling basic blocks play an important role in making inlining decisions, which is supported by Figure 7(a). It reports the number of function inlinings the FDO does when it uses a sampled profile,

\footnotetext{
${ }^{1}$ Without noting, the results in this and the following figures are similar across sampling rates, and the results at the lowest sampling rate are used.
} 


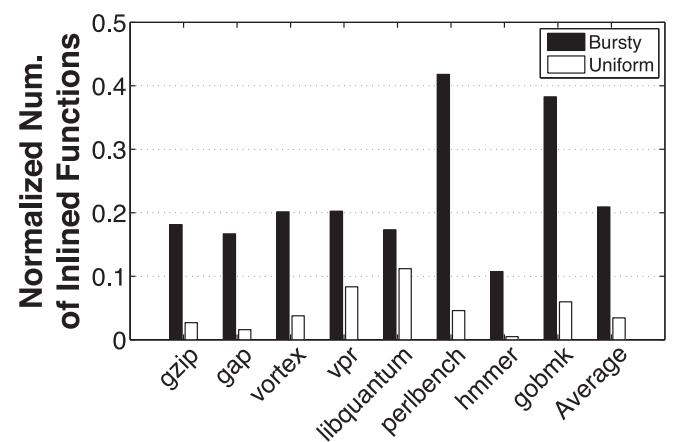

(a)

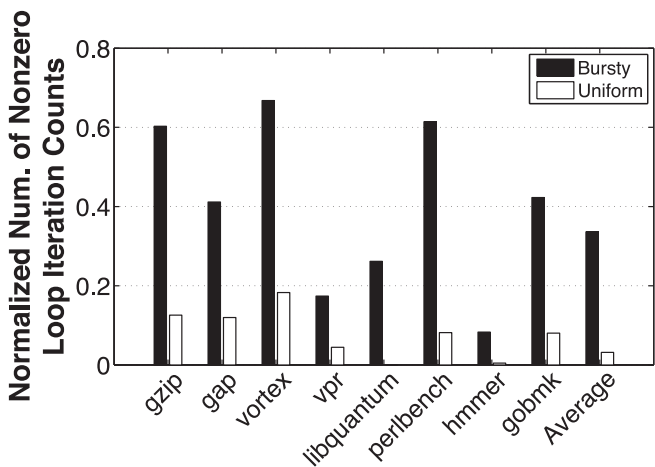

(b)

Fig. 7. (a) Normalized number of inlined functions. (b) Normalized number of loops having nonzero iteration counts.

normalized by the number when it uses the full profile. On average, the 0-counter errors cause the FDO to miss $79 \%$ inlining opportunities.

The second type of transformation, loop optimizations, also leverages profile information heavily. For example, in XLC, the iteration counts of a loop (in its one invocation) are calculated as $N_{\text {body }} / N_{\text {preheader }}$, where $N_{b o d y}$ is the value of a basic block counter in the loop body, and $N_{\text {preheader }}$ is that of the loop preheader. If a loop's preheader's counter value is 0 , its iteration count is annotated as "unknown." Since iteration count is one of the most important parameters in most loop transformations (e.g., loop versioning, loop unrolling, etc.), false information on the loop iteration count may seriously impair the transformation quality. However, because the loop preheader is usually executed much less frequently than its corresponding loop body, it is quite possible that although the sampler obtains a reasonable profile of the loop body, it cannot take advantage of it because of a zero counter value of the loop preheader. Figure 7(b) shows the percentage of loops that have "unknown" iteration counts when the sampled profile is used, while having a nonzero iteration counter when the exact profile is used. On average, only $33 \%$ of loops derived their iteration count information from the sampled profile. This percentage dropped to $3 \%$ for uniformly sampled profiles.

We now try to rectify the 0-counter errors to show their impact on performance. Figure 8 reports the results when the zero counters in the sampled profile are set to the values of their counterparts in the exact profile. That is, this rectification replaces the zero counters in the sampled profile with perfect information and hence completely removes the 0 -counter errors. We observe an increase of $14 \%$ and $23 \%$ performance improvement for bursty and uniform sampling, respectively. It echoes the results of basic block coverage and inlining decision difference, and shows that 0 -counter errors are one of the main sources leading to reduced FDO benefit.

3.1.2. Equality Errors. Zero-count errors mainly happen on cold events, while equality errors also happen on warm or hot events. An equality error refers to the case when the counters of two basic blocks in the same function have different values in the sampled profile but have the same value in the full profile.

For hot events, both sampling methods can get pretty good approximation of their values, which is reflected by the very high weighted accuracy reported in Section 2 . However, a decent approximation cannot prevent equality errors from happening.

To help quantify the amount of equality errors in the sampled profiles, we introduce a concept called consistency score. Let $G$ represent a group of basic blocks in an exact profile that have the same count (we denote the value as $v$ ) - that is, $\forall x \in G, \operatorname{count}(x)=v$. 


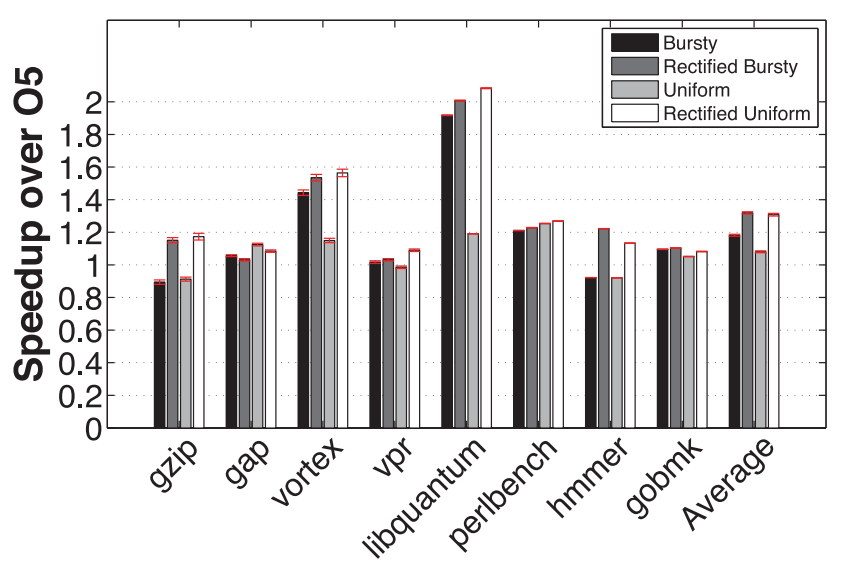

Fig. 8. Performance improvement after fixing 0-counter errors with exact profiles.

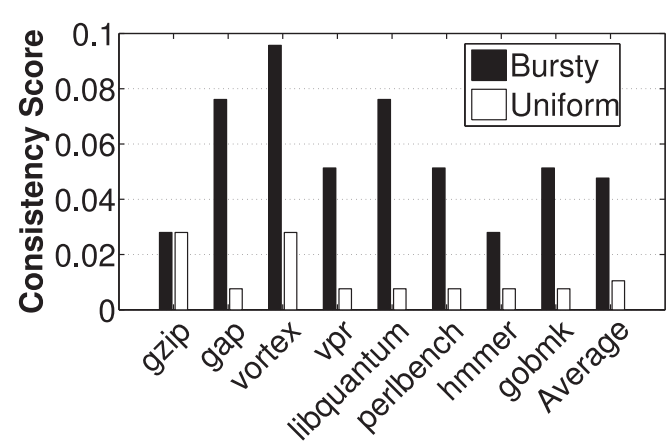

(a)

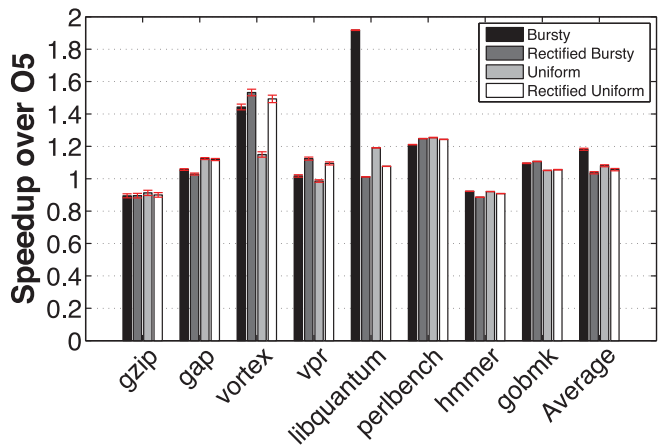

(b)

Fig. 9. (a) Consistency scores of all benchmarks. (b) Performance improvement after fixing equality errors with exact profiles.

Let $G^{\prime}$ be the largest subset of $G$ such that all members of $G^{\prime}$ have the same count (we denoted the value as $w$ ) - that is, $\forall x \in G^{\prime}, \operatorname{count}(x)=w$. Note, $v$ and $w$ do not have to equal each other. The consistency score of $G$ in the sampled profile is defined as $\frac{\left|G^{\prime}\right|}{|G|}$. An example is as follows. Suppose the original profile has seven basic blocks whose counts are as follows: $\operatorname{count}(\mathrm{A})=10, \operatorname{count}(\mathrm{B})=10, \operatorname{count}(\mathrm{C})=10, \operatorname{count}(\mathrm{D})=$ $10, \operatorname{count}(\mathrm{E})=10, \operatorname{count}(\mathrm{F})=20, \operatorname{count}(\mathrm{H})=20$. There would be two same-count groups: $\mathrm{G} 1=\{\mathrm{A}, \mathrm{B}, \mathrm{C}, \mathrm{D}, \mathrm{E}\}$, and $\mathrm{G} 2=\{\mathrm{F}, \mathrm{H}\}$. Suppose that the sampled profile has the counts as follows: $\operatorname{count}(\mathrm{A})=2, \operatorname{count}(\mathrm{B})=2, \operatorname{count}(\mathrm{C})=3, \operatorname{count}(\mathrm{D})=2, \operatorname{count}(\mathrm{E})=$ $3, \operatorname{count}(\mathrm{F})=7, \operatorname{count}(\mathrm{H})=7$. The largest subset of $\mathrm{G} 1$ that has the same count would be $\mathrm{G} 1^{\prime}=\{\mathrm{A}, \mathrm{B}, \mathrm{D}\}$, and the largest subset of G2 that has the same count would be G2' $=$ $\{\mathrm{F}, \mathrm{H}\}$. The consistency score of G1 would be $\frac{\left|G 1^{\prime}\right|}{|G 1|}=3 / 5=0.6$, and that of G2 would be $\frac{\left|G 2^{\prime}\right|}{|G 2|}=2 / 2=1$.

A consistency score must fall between 0 and 1 ; the higher it is, the better is the consistency preserved in the sampled profile. The overall consistency score of a sampled profile is just the average of the consistency scores of all the consistent groups in the corresponding exact profile.

Figure 9(a) reports the consistency scores of the sampled profiles. On average, the profiles have consistency scores of 0.47 and 0.01 for bursty and uniform sampling, 
respectively, suggesting that the sampling methods cannot preserve the consistency relation among counters well.

We study the potential performance gain by leveraging exact profiles to help rectify the equality errors in the sampled profiles. We identify all the basic block groups of each function in the exact profile that have the same counter value. Then, we set the counters in each group of the sampled profiles to their average. In this way, we maintain the equality relationship without changing the sampled profile's accuracy much. Figure 9(b) shows an improvement of up to 34\% for uniform sampling on vortex, demonstrating the large potential of fixing inconsistent basic block counters. For bursty sampling, we have an outlier libquantum, for which the rectification degrades the performance by $90 \%$. A plausible reason is that as the rectification is applied to its equality errors only, the rectified profile somehow forms some serious conflict with the zero-count errors remaining in the profile. Such an inference comes from an observation that the next subsection (Figure 16) will show: the degradation is completely reversed when zero-count errors are also fixed.

A detailed analysis shows that the primary influence of the equality errors is also on function inlining. The XLC compiler makes inlining decisions based on function hotness, size, and other factors. It first sorts calling edges based on their calling frequencies; a hotter call site gets a higher priority for inlining. If two functions have the same frequency, the smaller one has a higher priority. So, consider two functions, A and $\mathrm{B}$ (assuming $\mathrm{A}$ is much larger than $\mathrm{B}$ ), that have the same frequency in the exact profile but different in the sampled one (A has a larger frequency than B). The equality error may hence cause A rather than B to get inlined in the FDO on the sampled profile. As $\mathrm{A}$ is quite large, inlining it could cause many other functions to fail to get inlined because of the limit on the size of the resulting function. We observed a large degree of differences in inlining decisions of FDO before and after the equality errors are fixed, especially on programs vortex and vpr (details skipped for lack of space).

3.1.3. Inequality Errors. Complementary to equality errors are inequality errors, which refer to the case when one counter is greater than another in the sampled profile but much less so (or even smaller) in the full profile.

Our exploration starts with rectification of general inequality errors. The rectification algorithm ensures that the relative value relations among the counters in the sampled profile are the same as those in the full profile. For a function, it first gets a sorted (from small to large) list of the counters of the function, based on their values in the full profile. It then traverses the list and checks the values of the counters in the sampled profile. If it finds a counter, say, $B_{j}$, that has a value smaller than the value (denoted as $v$ ) of the counter right before it in the sorted list, it changes the value of $B_{j}$ to $v+\delta$, where $\delta$ is a predefined positive constant. When the algorithm gets to the end of the list, the counters in the whole sampled profile should have inequality relations that are consistent with those in the full profile.

We tried a spectrum of values for $\delta$, including $1,5,10$, and 20 . However, our results show that the rectification does not provide noticeable extra performance benefits over what the 0 -counter and equality rectifications can already provide.

Our analysis shows that it is not that inequality errors are unimportant, but that such rectification fails to capture the quantitative differences among basic block counters. The analysis is confirmed by our focused study on a special class of inequality errors, named branch bias errors.

Branch bias errors refer to the case when one of the two branches of a condition statement is taken substantially more often than the other branch in the full profile, but not that substantially often in the sampled profile. The condition statement is called an inconsistently biased statement. We classify condition statements into three 


\section{Branch Bias Ladder}

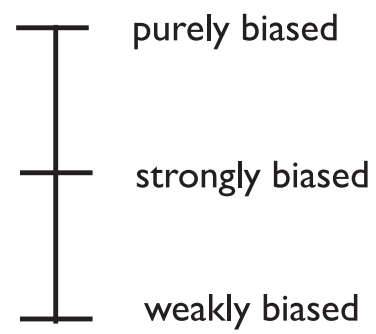

Fig. 10. Branch bias ladder. Going down the ladder is considered as bias errors.

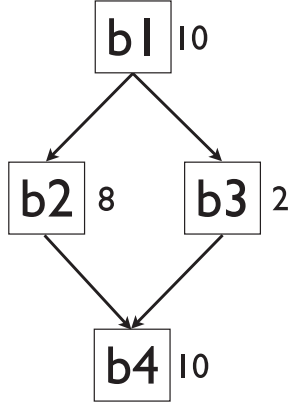

(a) full profile

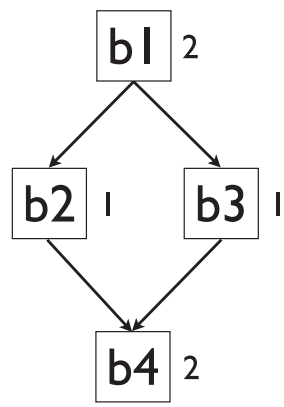

(b) sampled profile

Fig. 11. Example of branch bias errors.

categories based on their branch-taken rates in a profile: a condition statement is purely biased if only one of its branches has a greater-than-zero counter value, strongly biased if the counter value of one of its branches is no less than $80 \%$ of the counter value of the conditional statement, and weakly biased otherwise. They together form a three-level bias ladder as shown in Figure 10.

Compilers typically apply some more aggressive optimizations (e.g., code layout optimization) to a statement if it is at a higher level in the ladder. So in this work, we say that a branch bias error happens when the bias category of a condition statement in the sampled profile is at a level lower than its category in the full profile. Theoretically speaking, the converse cases may also affect the compilation; we, however, did not find strong evidence for it.

Figure 11 shows an example bias error: the condition statement is strongly biased in the full profile as the $b 1 \rightarrow b 2$ branch and $b 1 \rightarrow b 3$ branch of the condition statement have an $80 \%$ and $20 \%$ taken rate, respectively, but becomes weakly biased (with $50 \%$ and $50 \%$ taken rates) in the sampled profile.

Branch bias errors are common in the results of both bursty and uniform sampling. Figure 12(a) shows the percentage of the nonweakly biased branches in the full profile that remain nonweakly biased in the sampled profile. ${ }^{2}$ On average, only $54 \%$ and $18 \%$ remain consistent in the two profiles.

To quantify the influence of the branch bias errors, we rectify them by modifying the counters in the sampled profile such that the purely and strongly biased statements (in terms of their counter values in the full profile) fall into the same category as in the full

\footnotetext{
${ }^{2}$ We only deal with branches whose successors each have only one incoming edge; for them, branch-taken rates can be derived directly from the basic block counters.
} 


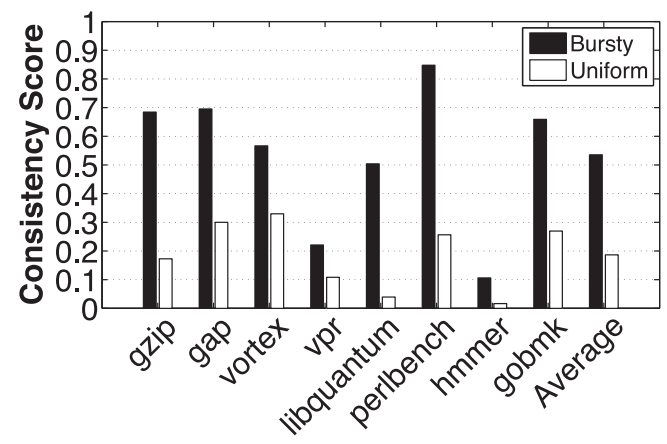

(a)

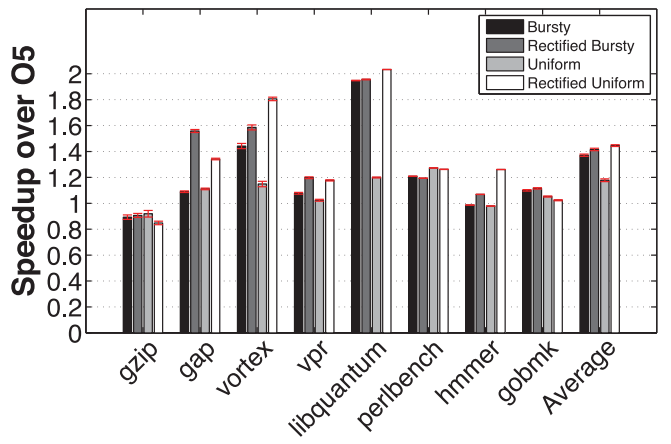

(b)

Fig. 12. (a) Percentage of consistently biased statements over the entire set of biased statements. (b) Performance improvement after fixing branch bias errors using exact profiles.

profile. Specifically, we raise the counter value of a branch that is strongly preferred in the full profile to $80 \%$ of the counter value of its control flow dominator in the sampled profile, and to $100 \%$ if the statement is purely biased in the full profile. We encounter cases where due to sampling effects, the dominator has a zero counter value while some of its branches have a positive value. We change the dominator counter value to 5 (the smallest number whose $20 \%$ remains an integer) before we apply the aforementioned rectification. The rectification goes in a top-down order based on the topology of statements in the control flow graph. It removes all the bias errors for the branches whose taken rates have been derived from the profiles.

Figure 12(b) shows the performance enhancement after the rectification. It gives substantial speedups on five out of the eight benchmarks. The largest speedup appears on libquantum due to the large influence of its conditional statements to its performance. On average, the rectification produces $5 \%$ and $26 \%$ speedups over all benchmarks for bursty sampling and uniform sampling, respectively.

3.1.4. Relations. We conduct experiments to examine the performance benefits when the three types of error rectifications are applied together. The results indicate that 0 -counter errors and bias errors complement each other. When being used together on all the benchmarks, they lead to average performance better than either can achieve.

However, when we add equality error rectification to them, we observe no noticeable changes in the performance. The reason is that equality error and bias error have some overlap: a purely biased condition statement also appears to have equality relations between its counter and the counter of one of its branches. So, rectification of bias errors may already rectify some of the equality errors. It should be noted that equality errors also cover equality relations among blocks beyond the scope of a conditional statement and its branches. However, the results suggest that errors in such relations are not essential for program optimizations. Figure 13 reports results of fixing both 0 -counter errors and branch bias errors. The average speedups are $14 \%$ and $23 \%$ more than without any fixing for bursty and uniform sampling, respectively.

3.1.5. Influence on Profile Accuracy. Sampling, by nature, can approximate frequent events well, which determines the accuracy of profiles. All three types of errors are caused by some subtle differences in values, and hence have only limited influence on the profile accuracy, as shown in Figure 14. That explains why there is no strong correlations between the accuracy of a profile and its usefulness for FDO, despite that the three types of profile errors affect FDO substantially. 


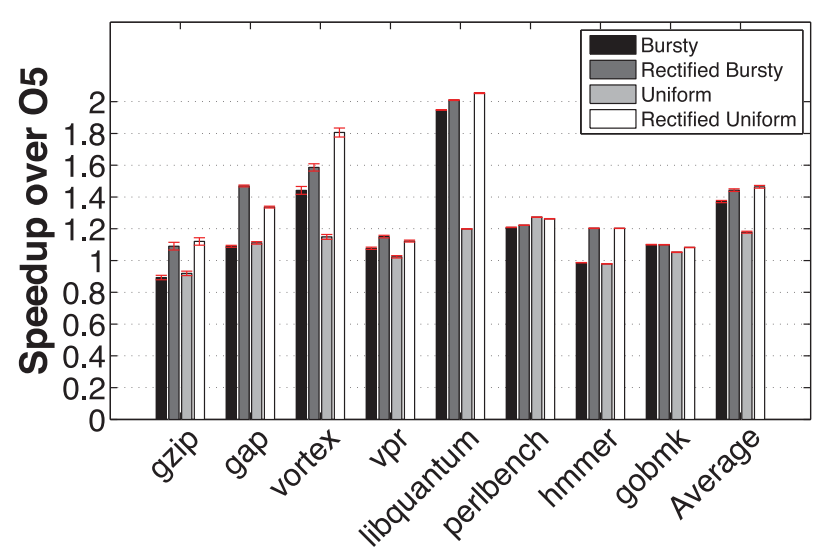

Fig. 13. Performance improvement after fixing 0-counter errors and branch bias errors using exact profiles.

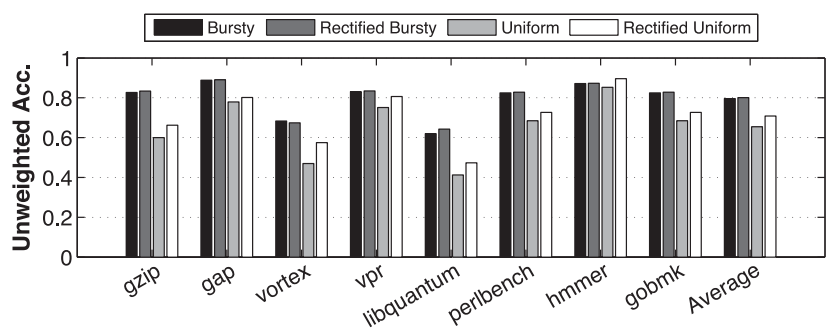

Fig. 14. Unweighted accuracy before and after profile error rectifications (ref input used).

\subsection{Simple Profile Rectification}

In the previous subsections, we analyzed the three critical types of sampling errors and showed the performance potential after fixing them with exact profiles. However, in reality, we do not have exact profiles in hand when recompiling the programs. We consider two alternative options.

The first is through static analysis. By purely analyzing the program, it tries to find out which basic blocks will be executed for sure, which basic blocks must have the same execution frequency, and which statements are strongly biased. There are also static approaches trying to predict the branches that are likely to be more frequently taken than other branches [Ball and Larus 1993; Wu and Larus 1994; Hashemi et al. 1997]. Some of these static analyses have already been exploited by default in the profiling process by XLC and GCC. For instance, the static compilation by XLC tries to find out straight lines of basic blocks, based on which, for profiling collection, it instruments only the first basic block in each straight line and infers the frequencies of other basic blocks automatically. This practice can already help avoid some equality errors: if it tried to sample all basic blocks, two basic blocks on a straight line could end up with different sample times. However, existing static methods are still subject to many limitations in rectifying profiles. For instance, Ball and Laurus acknowledged in their paper [Ball and Larus 1993] that static branch prediction is less accurate than profiling-based prediction. Moreover, the static method can predict which branch of a conditional statement is more often taken than the other branches of the conditional statement, but quantitative results are needed for rectifying the errors we described. For inequality errors, for instance, we would need to know whether it will be taken more than $80 \%$ of the time. The conservativeness of static analysis may also form some 


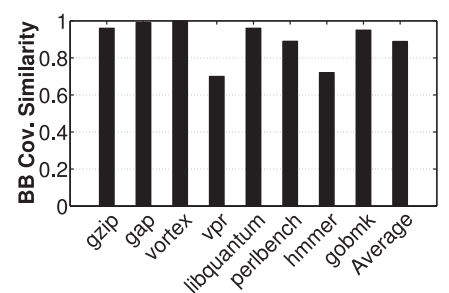

(a)

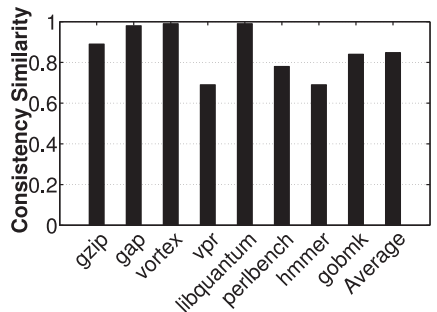

(b)

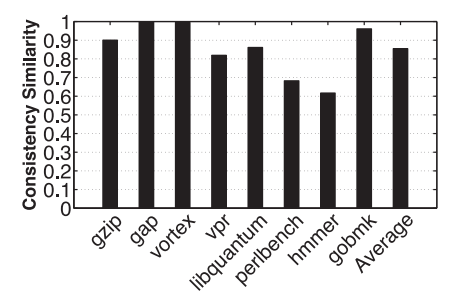

(c)

Fig. 15. (a) Similarity of basic block coverage patterns across ref inputs. (b) Similarity of basic block counter equality patterns across ref inputs. (c) Similarity of biased branch coverage across ref inputs.

barriers for the rectification. For two basic blocks that in practice almost always have the same counter values, static analysis cannot give such a conclusion if there is no way to prove that they must have the same counter values.

In this work, we choose a second option, which uses a training profile (on a smaller input) to rectify sampled profiles. For the observed relations among the three types of errors as mentioned in the previous section, the rectification focuses only on the 0 -counter and branch bias errors. For 0-counter errors, it assigns 1 to the counters of the basic blocks that are covered in the training profile but missed in the sampled profile. There are some other options, to use the exact counter value in the training profile or its scaled version. However, our experiments show that the minimal value change (from 0 to 1 ) is sufficient. For branch bias errors, the rectification is the same as the rectification mentioned in Section 3.1.3, except that the training profile rather than the exact profile is used.

An assumption underlying the design is that even though counter values change substantially across program executions on different inputs, their patterns relevant to those types of errors hold consistently across runs. Some earlier studies have shown some evidence of it [Fisher and Freudenberger 1992]. Our experiments echo the results. We use three ref inputs for each program to examine the assumption on all three types of errors. We quantify the basic block coverage pattern stableness by calculating the basic block coverage percentage for each pair of the three exact profiles and get their average, defined formally as follows:

$$
\left|C_{i} \cap C_{j}\right| / \max \left(\left|C_{i}\right|,\left|C_{j}\right|\right),
$$

where $C_{i}$ is the set of basic blocks covered in the $i$ th profile. We use the same formula, with $C_{i}$ redefined as the set of strongly and purely biased condition statements, to quantify the stableness of branch biases. To quantify the counter equality pattern stableness, we use the average consistency score between every pair of the ref profiles.

As Figure 15(a) shows, the basic block coverage among ref inputs is reasonably stable with a minimum of $70 \%$ and an average of $89 \%$. The counter equality pattern similarity and biased branch similarity are a bit less (on average, $85 \%$ and $87 \%$ ) but are still quite large.

3.2.1. Performance Results. We implement the rectification method by using the "train" and "ref" inputs included in the benchmark suite. The ref inputs are used in the testing runs. Because the ultimately intended usage of profile rectification is to rectify the errors in the sampled profiles collected at runtime in real (rather than training) runs, the rectification is applied to the sampled profiles on the ref runs. The full profiles collected on the train input of the benchmarks are used for the rectification.

We use the sampled profiles of the lowest sampling frequencies, which are 1/1000 for bursty sampling and 1/500000 for uniform sampling, for evaluation. As Figure 16 


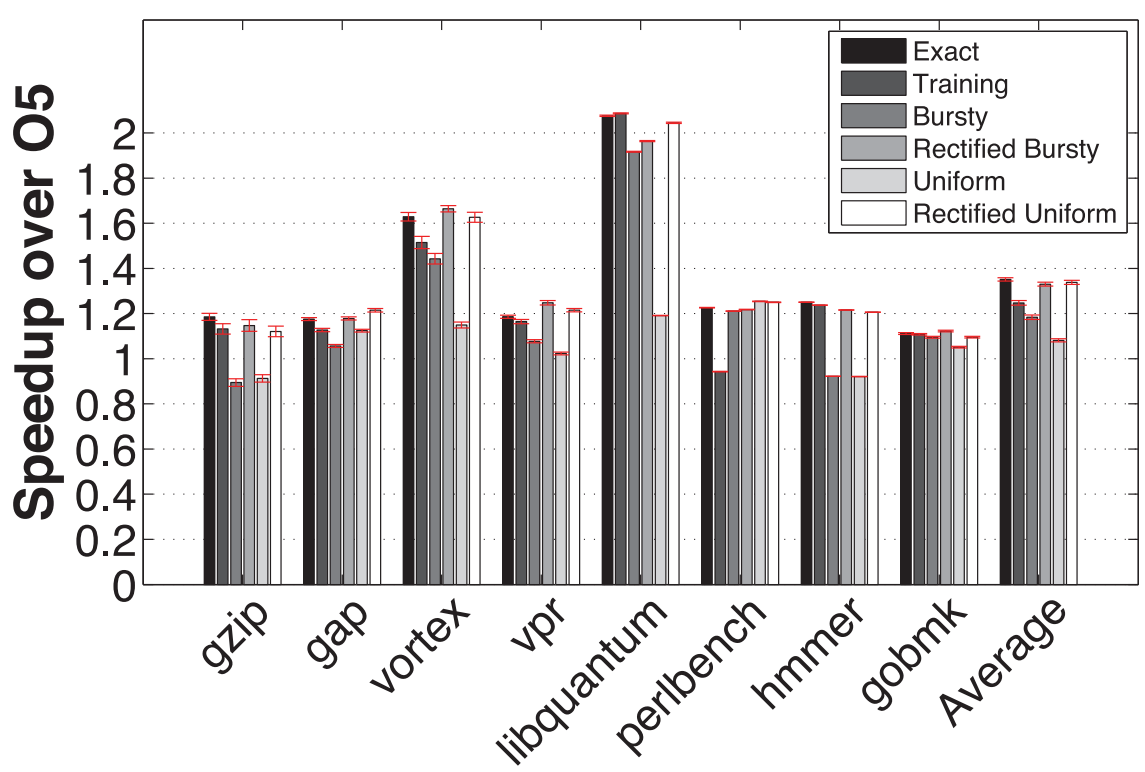

Fig. 16. FDO speedup based on exact profiles, training profiles, and rectified sampled profiles.

shows, the rectification achieves $98 \%$ of the full FDO benefit for both the bursty and uniform sampling cases. Compared to the sampled profiles, the rectified profiles enhance the FDO performance benefit by adding $15 \%$ and $26 \%$ extra speedups on average for bursty and uniform sampling, respectively.

An alternative to the profile rectification is just to use the training profile to do FDO. However, since the counter values may change dramatically across runs, the results are substantially inferior over the sampled profiles after rectification. As Figure 16 shows, on average, the rectified sampled profiles lead to around 8\% more speedup than which the training profiles produce. For the especially input-sensitive program perlbench, the training profile gives $6 \%$ slowdown, while the rectified profiles produce $1.21 \times$ and $1.25 \times$ speedup, showing the advantage of profile rectification over traditional offline profiling-based optimizations. It also suggests that with rectification, developers can keep using their simpler uniform sampling.

In Figure 16, there are several cases in which the exact profiles do not produce the best performance; the phenomenon persists in our repeated experiments. It indicates that the FDO implementation in the compilers can be improved to better leverage the profiling information. On the other hand, it is not a surprise given the large complexities in compiler construction (a similar, well-known demonstration of the complexity is that higher-level optimizations (e.g., “-O5”) do not always give better performance than lower-level optimizations (e.g., “-O3”)).

\subsection{Results from GCC}

The implementation of FDO in GCC differs much from that of XLC. For example, GCC does scaling before deciding whether to ignore a basic block or not. For each function, GCC finds the largest basic block counter value and sets it to a constant named BB_FREQ_MAX(10000), and other basic block counters values in this function are scaled accordingly. A basic block is ignored if its counter value is less than 1 after scaling. 


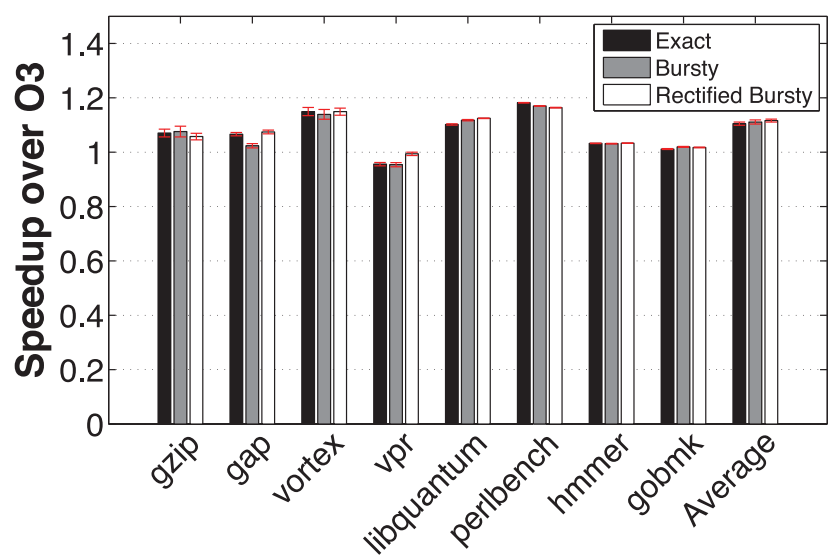

Fig. 17. Speedup on GCC. The baseline is by static compilation. The sampling bars show the performance of FDO after profile rectification.

Despite the different implementations between GCC and XLC, most of the insights reported on XLC hold on GCC. A prominent difference is that GCC tends to have a smaller degree of speedups by its FDO than XLC has. The reason probably comes from the less sophisticated design of its FDO implementation.

Figure 17 reports the speedups from a modified GCC v5.1 that enables bursty sampling with sampling rate $1 / 1000$. The settings include the cases of the highest static compilation, FDO on the exact profiles and rectified sampled profiles. The results show that the speedups from FDO are less prominent than those from the XLC compiler. However, if there is enough potential to improve, profile rectification can materialize most of the potential of FDO, which is best demonstrated on gap and vpr. Similar to the results from the XLC compiler, we observe higher speedups from bursty or rectified bursty sampling than the full profile in some cases, which once again indicates the potential for improvement of the FDO implementations in modern compilers.

The results in this section indicate that simple profile rectifications go a long way: despite the simplicity of the profile rectifications through training profiles, the rectified profiles - at even the lowest sampling rate-can help tap into most of the potential of FDO. Using a training profile helps explore the potential of profile rectification in this experiment, but after getting the insights that simple rectification to the two types of errors is sufficient, one may choose some other ways to do the rectification. For instance, one could combine sophisticated static program analysis with lightweight profiling on some ambiguous branches to identify the two kinds of value patterns of counters. Combined with cross-production run lightweight profiling [Tian et al. 2011], the method may provide more seamless integration with the JIT-based runtime engines. Detailed research in this direction is future work.

\section{DISCUSSIONS}

FDO is not only a part of the compilers for traditional imperative programming languages but also an important component of the managed runtime systems of Java and some scripting languages (e.g., Python, Ruby, JavaScript) that use Just-In-Time (JIT) compilers. The runtime systems typically profile the program execution during runtime and invoke JIT to optimize the programs based on the collected profiles. The online profiling is usually lightweight to be affordable. For example, Java virtual machines, including both HotSpot [Paleczny et al. 2001] and Jikes RVM [Arnold et al. 2000], typically do detailed profiling (e.g., collecting branch-taken frequencies) only during the 
interpretation (or basic compilation) stage; in other stages, they rely on timer-based sampling to collect profiles such as dynamic call graph. The relations between sampling errors and influence on program optimizations as revealed in this work could offer some insights for the design of JIT in these systems. However, it yet remains to be seen how the insights apply and whether different relations exist in those runtime scenarios.

This work offers the first systematic study on sampling errors and profile rectifications, but a comprehensive understanding of the influence of sampling errors on optimizations still needs further exploration. A future research direction is to extend this study to more types of profiles, such as branch target profiles, call target profiles, cache miss profiles, and so on.

\section{RELATED WORK}

Profiles have been used for program optimizations in many studies, ranging from code layout optimization [Pettis and Hansen 1990], to function inlining [Chang et al. 1992], to prefetching [Wu 2002], to reducing function indirection overhead, and so on. But relations between sampling errors and profile-driven optimizations have remained vaguely understood. The only study we have found directly on the relationship is by Langdale and Gross [2003]. In that study, the authors used only uniform sampling on machines a decade old. More importantly, the authors used compilers with quite preliminary FDO implementation: the full potential of the FDO on exact profiles is only about $3 \%$ speedup. Because of all these limitations, most conclusions from that work are out of date and even contrary to what we observe on modern compilers and machines (e.g., the results on busty sampling). The study presented in the this current article is an extension of the one presented recently [Wu et al. 2013]; they together form the first systematic study on the relations among sampling, profile accuracy, and profile usefulness on modern compilers, machines, and sampling methods. This study is the first that identifies the three critical classes of errors in program profiles incurred by sampling. It develops the first comprehensive method for automatic rectification of those errors.

Mytkowicz et al. [2010] have studied existing profilers and showed that they failed to agree with each other on the identification of hot functions. They found the sources of incorrectness and proposed a prototype of a random sampler to remove the biases in the previous implementations of random samplers.

There have been a number of earlier studies trying to estimate a program's dynamic profiles through static analysis [Ball and Larus 1993; Wu and Larus 1994; Hashemi et al. 1997]. A more recent work done by Lee et al. [2007] leverages a program's control flow constraints to improve the accuracy of dynamic call graphs from a sampled profile. As we discussed in Section 3.2, static analysis is subject to some major limitations for rectifying the kinds of errors explored in this work. However, combining static analysis with the dynamic method for profile rectification is worth exploring in the future.

A number of studies have focused on effective instrumentation or sampling. Knuth and Stevenson [1973] show that they need to instrument only a minimum number of edges of the control flow graph and calculate the counters for all other edges in an offline analysis. The technique has helped save profiling overhead for modern compilers. Ball and Larus [1996] propose an efficient path profiling technique, which encodes each path into a nonnegative integer and uses it as an index to update global counters efficiently. Vaswani et al. [2007] separate interesting paths and profile them with low overhead. Bond and McKinley [2005] point out that Ball's path profiling algorithm overhead bottleneck is the counter update and uses sampling to reduce the overhead to enable continuous profiling. Arnold and Ryder [2001] reduce instrumentation overhead of a Java JIT compilation system by creating a fully instrumented copy for each 
method and periodically switching execution to that copy to collect profile information. Buytaert et al. [2007] report that timer-based sampling has several limitations and propose to enhance it through hardware performance monitors. Chen et al. [2010] construct edge profiles from hardware counters and use the constructed profiles to direct recompilation.

A number of works have studied the influence of different inputs on program profiles and optimizations. Kim and others have proposed 2D profiling to detect inputdependent branches with a single input dataset [Kim et al. 2006]. Berube and others have studied methods to combine profiles from different runs of a program [Berube et al. 2011]. Eeckhout et al. [2003] have used statistical approaches to quantify the impact of input datasets on program behaviors. Tian and others have proposed inputcentric program optimizations trying to bring program inputs into the focus of program analysis and optimizations [Tian et al. 2010; Jiang et al. 2010].

\section{CONCLUSION}

This article presents a systematic exploration on the relations among sampling rates, profile accuracy, and profile usefulness for FDO. The exploration covers seven factors in four levels. It reveals some counterintuitive relations, the most prominent of which are that higher sampling rates (within a typical sample rate range) do not lead to more accurate profiles when uniform sampling is used, and more importantly, no matter which sampling method is used, the accuracy of the profiles does not show a strong correlation with their usefulness for FDO. The article then describes a detailed analysis on three important types of errors caused by sampling: 0-counter errors, equality errors, and inequality errors. Among various inequality errors, it identifies an especially important type, namely, branch bias errors. It finds out that most of the influence of equality errors is subsumed by that of branch bias errors.

Based on empirically confirmed cross-input stableness of relevant value patterns in profiles, the article then presents a simple way to rectify 0 -counter errors and branch bias errors. The dramatic enhancement of the FDO benefits concludes that simple rectification of the two types of errors in a profile is promising in tapping into the full potential of FDO. It also suggests that with the simple profile rectifications, sampling rate (and hence sample overhead) can be significantly lowered without hurting the FDO benefits. The work provides the first principled understanding in effective collection of profiles for FDO and may help remove the barriers for practical adoptions of FDO.

\section{REFERENCES}

M. Arnold, S. Fink, D. Grove, M. Hind, and P. F. Sweeney. 2000. Adaptive optimization in the JalapeNO JVM. In Proceedings of the 15th ACM SIGPLAN Conference on Object-Oriented Programming, Systems, Languages, and Applications.

M. Arnold and D. Grove. 2005. Collecting and exploiting high-accuracy call graph profiles in virtual machines. In Proceedings of the International Symposium on Code Generation and Optimization.

M. Arnold and B. G. Ryder. 2001. A framework for reducing the cost of instrumented code. In Proceedings of ACM SIGPLAN Conference on Programming Language Design and Implementation. Snowbird, Utah.

T. Ball and J. R. Larus. 1993. Branch prediction for free. In Proceedings of the ACM SIGPLAN 1993 Conference on Programming Language Design and Implementation (PLDI'93).

T. Ball and J. R. Larus. 1996. Efficient path profiling. In Proceedings of the 29th Annual ACM/IEEE International Symposium on Microarchitecture.

P. Berube, A. Preuss, and J. N. Amaral. 2011. Combined profiling: Practical collection of feedback information for code optimization. In Proceedings of the 2nd ACM/SPEC International Conference on Performance Engineering.

M. D. Bond and K. S. McKinley. 2005. Continuous path and edge profiling. In Proceedings of the 29th Annual ACM/IEEE International Symposium on Microarchitecture. 
D. Buytaert, A. Georges, M. Hind, M. Arnold, L. Eeckhout, and K. De Bosschere. 2007. Using HPM-sampling to drive dynamic compilation. In Conference on Object-Oriented Programming, Systems, Languages, and Applications (OOPSLA'07).

P. P. Chang, S. A. Mahlke, W. Y. Chen, and W. Hwu. 1992. Profile-guided automatic inline expansion for C programs. Software Practice \& Experience 22, 5 (1992), 349-369.

D. Chen, N. Vachharajani, R. Hundt, S. Liao, V. Ramasamy, P. Yuan, W. Chen, and W. Zheng. 2010. Taming hardware event samples for FDO compilation. In Proceedings of the 8th Annual IEEE/ACM International Symposium on Code Generation and Optimization. New York, NY.

L. Eeckhout. 2010. Computer architecture performance evaluation methods. Synthesis Lectures on Computer Architecture (2010).

L. Eeckhout, H. Vandierendonck, and K. De Bosschere. 2003. Quantifying the impact of input data sets on program behavior and its applications. Journal of Instruction-Level Parallelism 5 (2003), 1-33.

J. A. Fisher and S. M. Freudenberger. 1992. Predicting conditional branch directions from previous runs of a program. In Proceedings of the 5th International Conference on Architectural Support for Programming Languages and Operating Systems (ASPLOS'92).

A. H. Hashemi, D. R. Kaeli, and B. Calder. 1997. Procedure mapping using static call graph estimation. In Workshop on Interaction between Compiler and Computer Architecture.

M. Hirzel and T. M. Chilimbi. 2001. Bursty tracing: A framework for low-overhead temporal profiling. In Proceedings of ACM Workshop on Feedback-Directed and Dynamic Optimization.

Y. Jiang, E. Z. Zhang, K. Tian, F. Mao, M. Gethers, and X. Shen. 2010. Exploiting statistical correlations for proactive prediction of program behaviors. In International Symposium on Code Generation and Optimization (CGO'10).

H. Kim, M. Aater Suleman, O. Mutlu, and Y. N. Patt. 2006. 2D-profiling: Detecting input-dependent branches with a single input data set. In Proceedings of the International Symposium on Code Generation and Optimization.

D. Knuth and F. Stevenson. 1973. BIT Numerical Mathematics 13, 3 (1973), 313-322.

G. Langdale and T. Gross. 2003. Evaluating the relationship between the usefulness and accuracy of profiles. In Proceedings of the Workshop on Duplicating, Deconstructing, and Debunking.

B. Lee, K. Resnick, M. D. Bond, and K. S. Mckinley. 2007. Correcting the dynamic call graph using control flow constraints. In International Conference on Compiler Construction.

H. Mousa and C. Krintz. 2005. HPS: Hybrid profiling support. In Proceedings of the 14th International Conference on Parallel Architectures and Compilation Techniques.

T. Mytkowicz, A. Diwan, M. Hauswirth, and P. F. Sweeney. 2010. Evaluating the accuracy of Java profilers. In Proceedings of the 2010 ACM SIGPLAN Conference on Programming Language Design and Implementation. New York, NY.

M. Paleczny, C. Vick, and C. Click. 2001. The Java hotspotTM server compiler. In Proceedings of the 2001 Symposium on JavaTM Virtual Machine Research and Technology Symposium - Volume 1.

K. Pettis and R. C. Hansen. 1990. Profile guided code positioning. In Proceedings of the ACM SIGPLAN Conference on Programming Language Design and Implementation.

X. Shen, J. Shaw, B. Meeker, and C. Ding. 2007. Locality approximation using time. In Proceedings of the 34th Annual ACM SIGPLAN-SIGACT Symposium on Principles of Programming Languages.

K. Tian, Y. Jiang, E. Zhang, and X. Shen. 2010. An input-centric paradigm for program dynamic optimizations. In the Conference on Object-Oriented Programming, Systems, Languages, and Applications (OOPSLA'10).

K. Tian, E. Zhang, and X. Shen. 2011. A step towards transparent integration of input-consciousness into dynamic program optimizations. In the Conference on Object-Oriented Programming, Systems, Languages, and Applications (OOPSLA).

K. Vaswani, A. V. Nori, and T. M. Chilimbi. 2007. Preferential path profiling: Compactly numbering interesting paths. In Proceedings of the 34th Annual ACM SIGPLAN-SIGACT Symposium on Principles of Programming Languages.

B. Wu, M. Zhou, X. Shen, Y. Gao, R. Silvera, and G. Yiu. 2013. Simple profile rectifications go a long way. In Proceedings of the European Conference on Object-Oriented Programming. 654-678.

Y. Wu. 2002. Efficient discovery of regular stride patterns in irregular programs. In Proceedings of ACM SIGPLAN Conference on Programming Language Design and Implementation.

Y. Wu and J. R. Larus. 1994. Static branch frequency and program profile analysis. In Proceedings of the 27th Annual International Symposium on Microarchitecture (MICRO’94).

Received March 2015; revised November 2015; accepted November 2015 